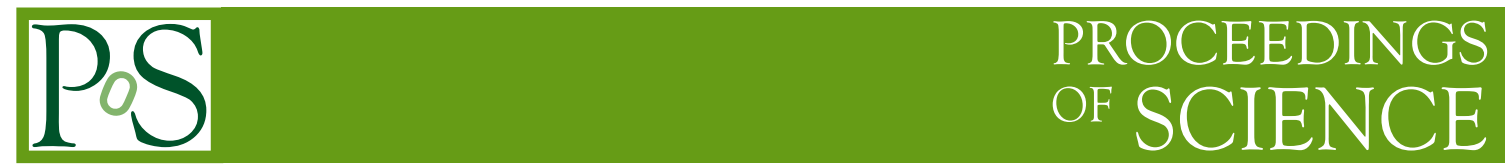

\title{
Recent experimental results from the SND detector
}

\author{
A.A. Korol ${ }^{* a b c}$ and M.N. Achasov ${ }^{a b}$ and A.Yu. Barnyakov ${ }^{a b}$ and K.I.Beloborodov ${ }^{a b}$ \\ and A.V.Berdyugin ${ }^{a b}$ and A.G. Bogdanchikov ${ }^{a}$ and A.A.Botov ${ }^{a}$ and V.P. Druzhinin ${ }^{a b}$ \\ and V.B. Golubev ${ }^{a b}$ and L.V. Kardapoltsev ${ }^{a b}$ and A.G. Kharlamov ${ }^{a b}$ and \\ S.V. Koshuba ${ }^{a b}$ and L.A. Korneev ${ }^{a}$ and D.P. Kovrizhin ${ }^{a b}$ and A.S. Kupich ${ }^{a b}$ and \\ R.A. Litvinov ${ }^{a}$ and K.A. Martin ${ }^{a b}$ and N.A. Melnikova ${ }^{a b}$ and A.E. Obrazovsky ${ }^{a}$ and \\ E.V. Pakhtusova ${ }^{a}$ and K.V. Pugachev ${ }^{a b}$ and S.I. Serednyakov ${ }^{a b}$ and D.A. Shtol ${ }^{a}$ and \\ Z.K. Silagadze ${ }^{a b}$ and I.K. Surin ${ }^{a}$ and Yu.V. Usov ${ }^{a b}$ and A.V. Vasiljev ${ }^{a b}$ and \\ V.N. Zhabin ${ }^{a b}$ and V.V. Zhulanov ${ }^{a b}$ \\ ${ }^{a}$ Budker Institute of Nuclear Physics, Novosibirsk, Russia \\ ${ }^{b}$ Novosibirsk State University, Novosibirsk, Russia \\ ${ }^{c}$ E-mail: A.A.Koroleinp.nsk.su
}

\begin{abstract}
We present recent experimental results for e+e annihilation into hadrons below $2 \mathrm{GeV}$ obtained with the SND detector at the VEPP-2000 collider. The analyses are based on data collected in the detector runs from 2010 to 2017. During the period from 2014 to 2016 collider and detector underwent significant upgrades which allowed an increase in luminosity and detector efficiency. In particular, results of studying $e^{+} e^{-} \rightarrow \pi^{+} \pi^{-}, \eta \rightarrow e^{+} e^{-}, e^{+} e^{-} \rightarrow n \bar{n}, e^{+} e^{-} \rightarrow K^{+} K^{-} \eta$, $e^{+} e^{-} \rightarrow \pi^{+} \pi^{-} \eta, e^{+} e^{-} \rightarrow K_{S} K_{L} \pi^{0}, e^{+} e^{-} \rightarrow \pi^{+} \pi^{-} \pi^{0} \eta, e^{+} e^{-} \rightarrow \omega \pi^{0}, e^{+} e^{-} \rightarrow \pi^{0} \gamma$, and $e^{+} e^{-} \rightarrow \pi^{+} \pi^{-} 4 \pi^{0}$ reactions are considered in this paper.
\end{abstract}

XIII Quark Confinement and the Hadron Spectrum - Confinement 2018

31 July - 6 August 2018

Maynooth University, Ireland

${ }^{*}$ Speaker. 


\section{Introduction}

The SND [ [ $[$, [] is a general purpose nonmagnetic detectors installed at the interaction region of the VEPP-2000 collider [3]]. The collider can operate in c.m. energies $0.3-2.0 \mathrm{GeV}$.

The detector includes a spherical electromagnetic calorimeter, a cylindrical tracking system, a threshold Cherenkov counters and a cosmic events veto detector. The electromagnetic calorimeter covers $0.95 \times 4 \pi$ of the solid angle, has $13.4 X_{0}$ thickness, has an energy resolution $\frac{\delta E}{E} \sim \frac{4.2 \%}{\sqrt[4]{E(\mathrm{GeV})}}$ and angle resolution $\delta \varphi=\frac{0.82^{\circ}}{\sqrt{E(\mathrm{GeV})}} \oplus 0.63^{\circ}$. The tracking system covers $0.94 \times 4 \pi$ of the solid angle, and has an angular resolution $\delta \varphi=0.55^{\circ}, \delta \theta=1.2^{\circ}$ [䧃]. Cherenkov counters allows $K / \pi$ separation for $E<1 \mathrm{GeV}$.

Accurate VEPP-2000 beam energy measurement is provided by a system based on Compton backscattered laser photons [[5].

During 2010-2013 about $70 \mathrm{pb}^{-1}$ of integrated luminosity was recorded in the energy range $0.3-2.0 \mathrm{GeV}$. During this period the luminosity was limited by the positrons shortage. That's why from 2014 until 2016 the collider and detectors underwent a significant upgrade. The experiments were restarted by the end of 2016 [6] with a more intensive positron source. About $120 \mathrm{pb}^{-1}$ of integrated luminosity has been accumulated during 2017 and 2018 years (Fig.W).

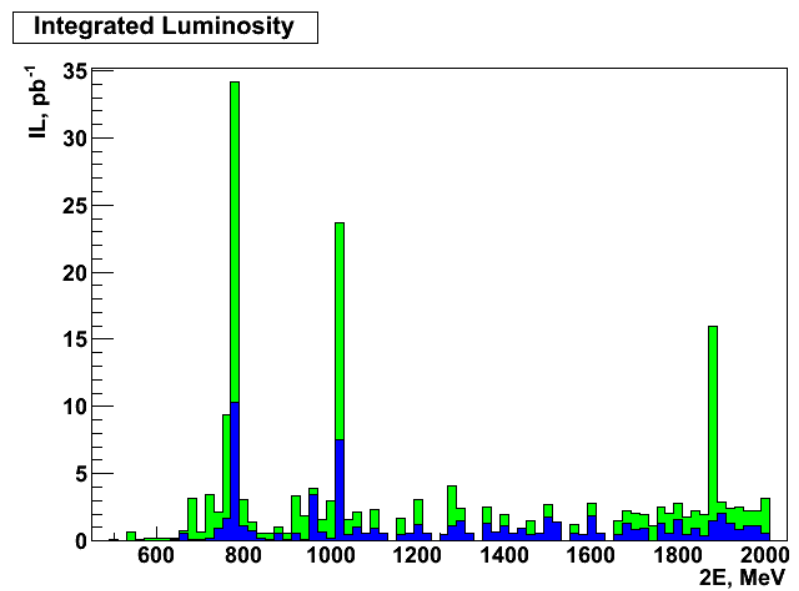

Figure 1: Distribution of accumulated luminosity over the c.m.energy for 2010-2013(blue) and 2017-2018(green) periods of data taken

The main goal of SND detector experiments is the measurement of exclusive hadronic cross sections below $2 \mathrm{GeV}$. This is important in order to calculate the hadron contribution to the vacuum polarisation (muon $(g-2)$ and running $\left.\alpha_{Q E D}\right)$.

\section{Experimental results}

Process $e^{+} e^{-} \rightarrow \pi^{+} \pi^{-}$

The dominant contribution to the ratio of the hadronic cross section to the muon cross section in electron-positron collisions $\mathrm{R}(\mathrm{s})$ in the c.m. energy range $\sqrt{s}<1 \mathrm{GeV}$ comes from the $e^{+} e^{-} \rightarrow$ 
$\pi^{+} \pi^{-}$mode. A preliminary result on the form factor obtained at SND is shown in Fig.2. The 3 layer structure of calorimeters gives the possibility to separate electrons and pions [ $[\mathbf{]}]$. The contribution from $e^{+} e^{-} \rightarrow \mu^{+} \mu^{-}$was determined from the theoretical cross section and subtracted. The systematic error is $0.9 \%$ for the energy range $\sqrt{s}<0.6 \mathrm{GeV}$ and $0.8 \%$ for $\sqrt{s}>0.6 \mathrm{GeV}$. The comparison of SND result with previous measurements at BABAR[8] and KLOE[Q] detectors is shown in Fig.2b. In the energy range $\sqrt{s}<0.6 \mathrm{GeV}$ there is a difference with the BABAR results of about $3 \%$.
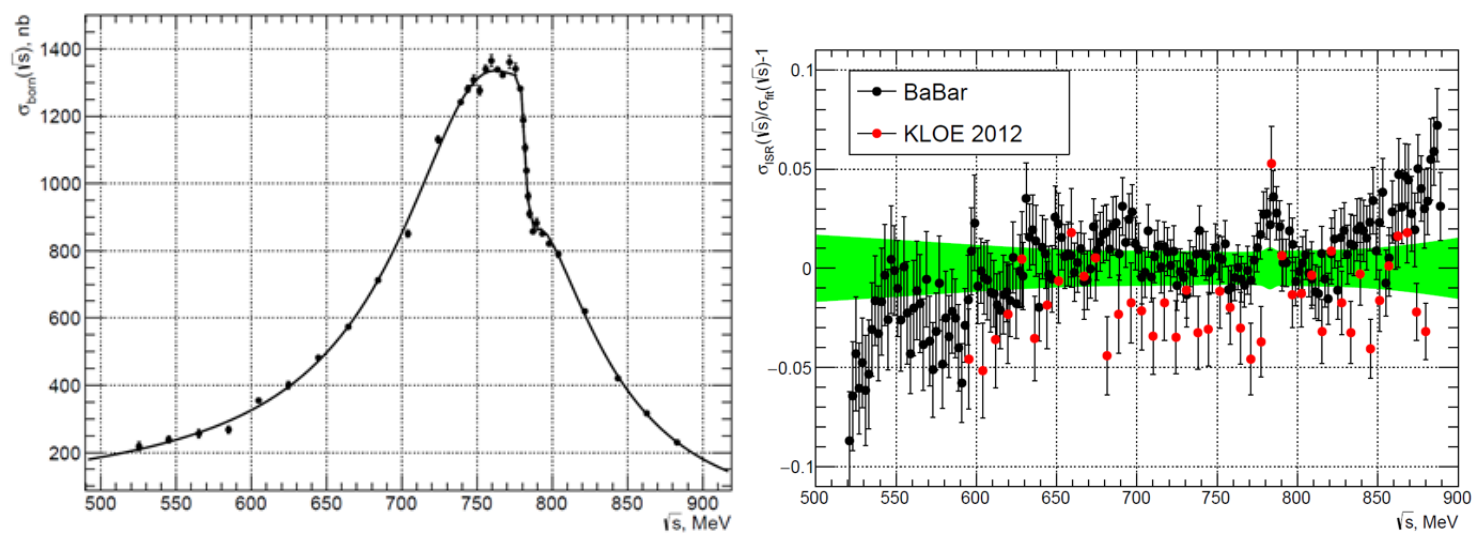

(a) The cross section of the $e^{+} e^{-} \rightarrow \pi^{+} \pi^{-}$process (b) Comparison of SND results with BABAR [8] and obtained at SND.

KLOE[Q], green band is for SND cross section systematic errors

Figure 2

\section{Search for $\eta \rightarrow e^{+} e^{-}$decay}

Because of the small probability the pseudoscalar meson decays $P \rightarrow l^{+} l^{-}$(here $l=e, \mu$ ) are sensitive to contributions that are not described in the framework of the Standard Model. The unitary limit for this decay is $\mathscr{B}^{\mathrm{UL}}\left(\eta \rightarrow e^{+} e^{-}\right)=1.78 \times 10^{-9}$.

Since the visible $e^{+} e^{-} \rightarrow \eta$ cross section is proportional to the branching fraction $\mathscr{B}(\eta \rightarrow$ $e^{+} e^{-}$), the search was carried out using $e^{+} e^{-} \rightarrow \eta \rightarrow \pi^{0} \pi^{0} \pi^{0}$ process. No candidate events for the process has been found. An upper limit has been set [ए]] $\mathscr{B}\left(\eta \rightarrow e^{+} e^{-}\right)<7 \times 10^{-7}$ at the $90 \%$ confidence level.

Process $e^{+} e^{-} \rightarrow n \bar{n}$

The cross section for the $e^{+} e^{-} \rightarrow n \bar{n}$ process was measured by SND using new data obtained in 2017. The preliminary result in comparison with previous SND data is shown in Fig. B]. The difference with our previous result is related to inaccurate $n \bar{n} \mathrm{MC}$ simulation and inaccurate beam and cosmic backgrounds estimation in earlier measurement. The systematic uncertainty is estimated to be about $20 \%$ mainly due to MC simulation. 


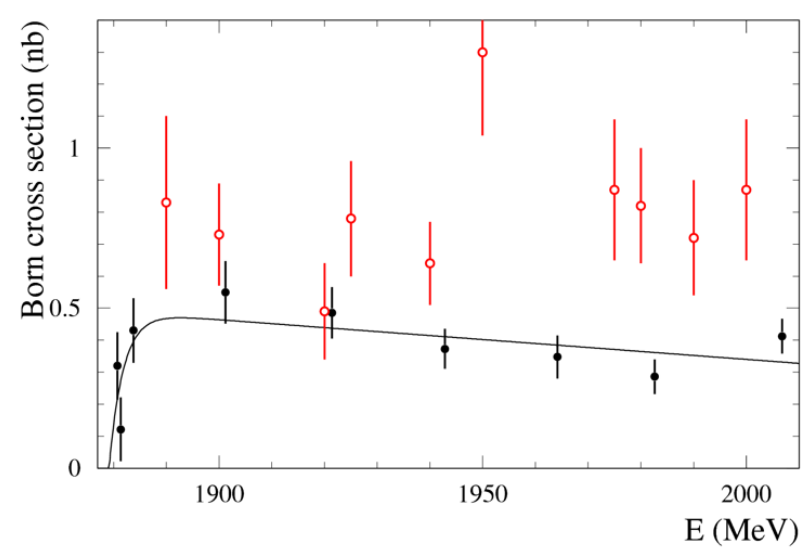

Figure 3: The $e^{+} e^{-} \rightarrow n \bar{n}$ cross section measured by SND using new data obtained in 2017 (black filled circles) in comparison with previous SND measurement[[]] (red empty circles)

\section{Process $e^{+} e^{-} \rightarrow K^{+} K^{-} \eta$}

This process is studied in the $\eta \rightarrow \gamma \gamma$ decay mode. The $\pi / K$ separation was done using the drift chamber ionization and the aerogel counter data. The measured cross section [12], in comparison with BABAR data [[3]], is shown in Fig. 6 . The fit to the cross section data is performed under the hypothesis that the main mechanism of this reaction is $e^{+} e^{-} \rightarrow \phi(1680) \rightarrow \phi(1020) \eta$.

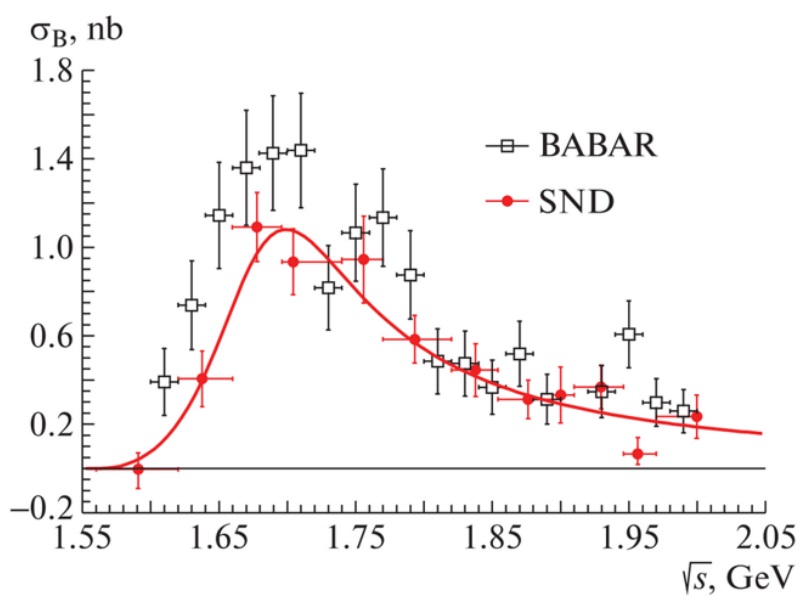

Figure 4: The $e^{+} e^{-} \rightarrow K^{+} K^{-} \eta$ cross section measured by SND at VEPP-2000 [ए2] and in the BABAR experiment [ए3]]. The solid curve is the result of the fit described in the text.

Process $e^{+} e^{-} \rightarrow \pi^{+} \pi^{-} \eta$

The cross section for the $e^{+} e^{-} \rightarrow \pi^{+} \pi^{-} \eta$ process was studied in the $\eta \rightarrow 3 \pi^{0}$ decay mode[ [प4]]. The result agrees with the previous SND measurement in the $\eta \rightarrow 2 \gamma$ decay mode[ [प5] (Fig. [D). The fit of the cross section was performed using a sum of the $\rho(770), \rho(1450)$ and $\rho(1700)$ contributions. The phases obtained in this fit are equal to $\phi_{\rho(1450)}=\pi$ and $\phi_{\rho(1700)}=\pi$ while the quark model suggest it to be opposite in sign [ए6]. 


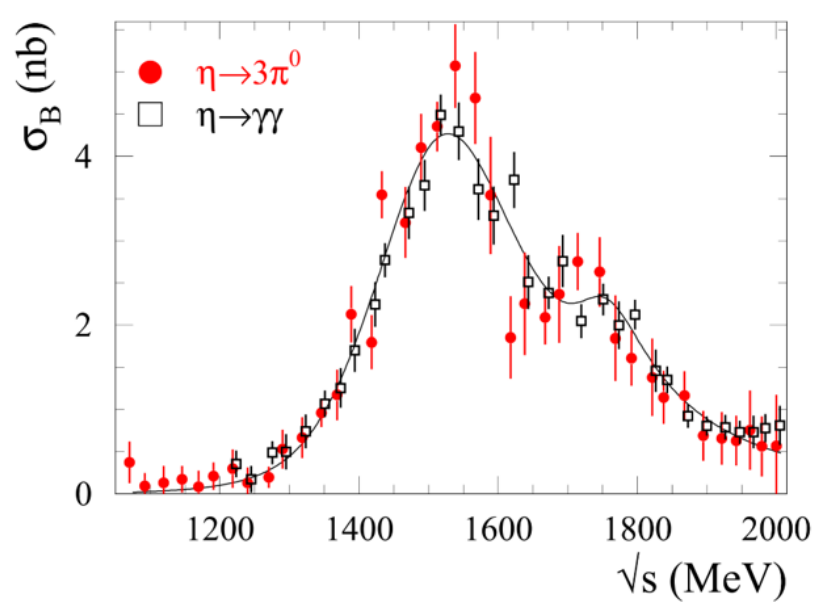

Figure 5: The $e^{+} e^{-} \rightarrow \pi^{+} \pi^{-} \eta$ cross section measured by SND at VEPP-2000 in the $\eta \rightarrow 3 \pi^{0}$ and $\eta \rightarrow 2 \gamma$ modes. The solid curve is the result of the fit to a sum of the $\rho(770), \rho(1450)$ and $\rho(1700)$ contributions.

Process $e^{+} e^{-} \rightarrow K_{S} K_{L} \pi^{0}$

The process $e^{+} e^{-} \rightarrow K_{S} K_{L} \pi^{0}$ is one of three charged modes of the process $e^{+} e^{-} \rightarrow K \bar{K} \pi$, which gives a notable contribution to the total cross section of $e^{+} e^{-}$annihilation into hadrons in the center-of-mass energy range $\sqrt{s}=1.5-1.8 \mathrm{GeV}$. It is also important for spectroscopy of $s \bar{s}$ vector meson states. From these states, only the lowest $\phi(1020)$ is well studied, and its branching fractions are measured down to $10^{-5}$. Spectroscopy of the first excited state $\phi(1680)$ is far from completion. Its main decay mode is $\phi(1680) \rightarrow K^{*}(892) K$ with the $K^{*}(892) \rightarrow K \pi$. We study the neutral subprocess $e^{+} e^{-} \rightarrow K_{S} K_{L} \pi^{0}$ in $K_{S} \rightarrow \pi^{0} \pi^{0}$ decay mode. The obtained cross section [[17] in comparison with BABAR data [18] and predictions from $K K^{*}$ isospin relations[[13] is shown in Fig. 目.

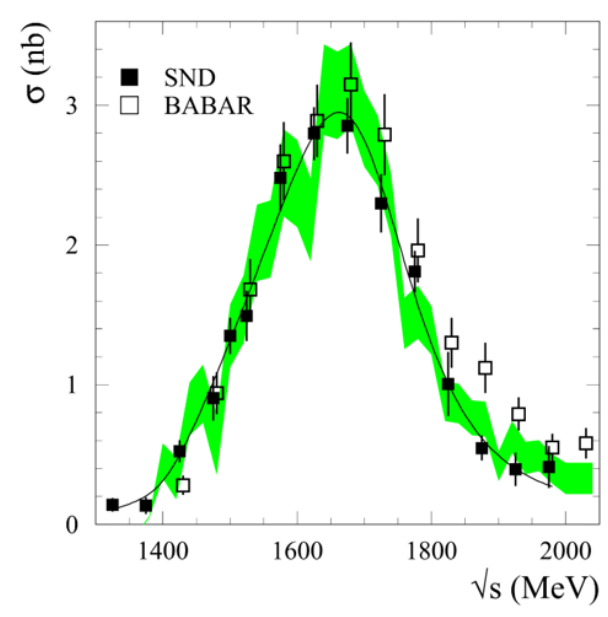

Figure 6: The $e^{+} e^{-} \rightarrow K_{S} K_{L} \pi^{0}$ cross section measured by SND at VEPP-2000 [प]] in comparison with BABAR data[[18]. The green band represents the predictions from $K K^{*}$ isospin relations [[1]]]. The solid curve is the result of the fit to SND data with the VMD model. 
Process $e^{+} e^{-} \rightarrow \pi^{+} \pi^{-} \pi^{0} \eta$

The process $e^{+} e^{-} \rightarrow \pi^{+} \pi^{-} \pi^{0} \eta$ has complex internal structure. Our preliminary study show that there are at least four mechanisms for this reaction: $\omega \eta, \phi \eta, a_{0}(980) \rho$, and structureless $\pi^{+} \pi^{-} \pi^{0} \eta$. The known $\omega \eta$ and $\phi \eta$ contributions explain about $50-60 \%$ of the cross section below $1.8 \mathrm{GeV}$. Above $1.8 \mathrm{GeV}$ the dominant mechanism is $a_{0} \rho$. The preliminary result on the total $e^{+} e^{-} \rightarrow \pi^{+} \pi^{-} \pi^{0} \eta$ cross section in comparison with CMD-3 data[प्प] is shown in Fig. [a. The cross section for the subprocess $e^{+} e^{-} \rightarrow \omega \eta$ is measured separately [ㅁ] and shown in Fig. comparison with the BABAR and CMD-3 measurements [2], [9]].
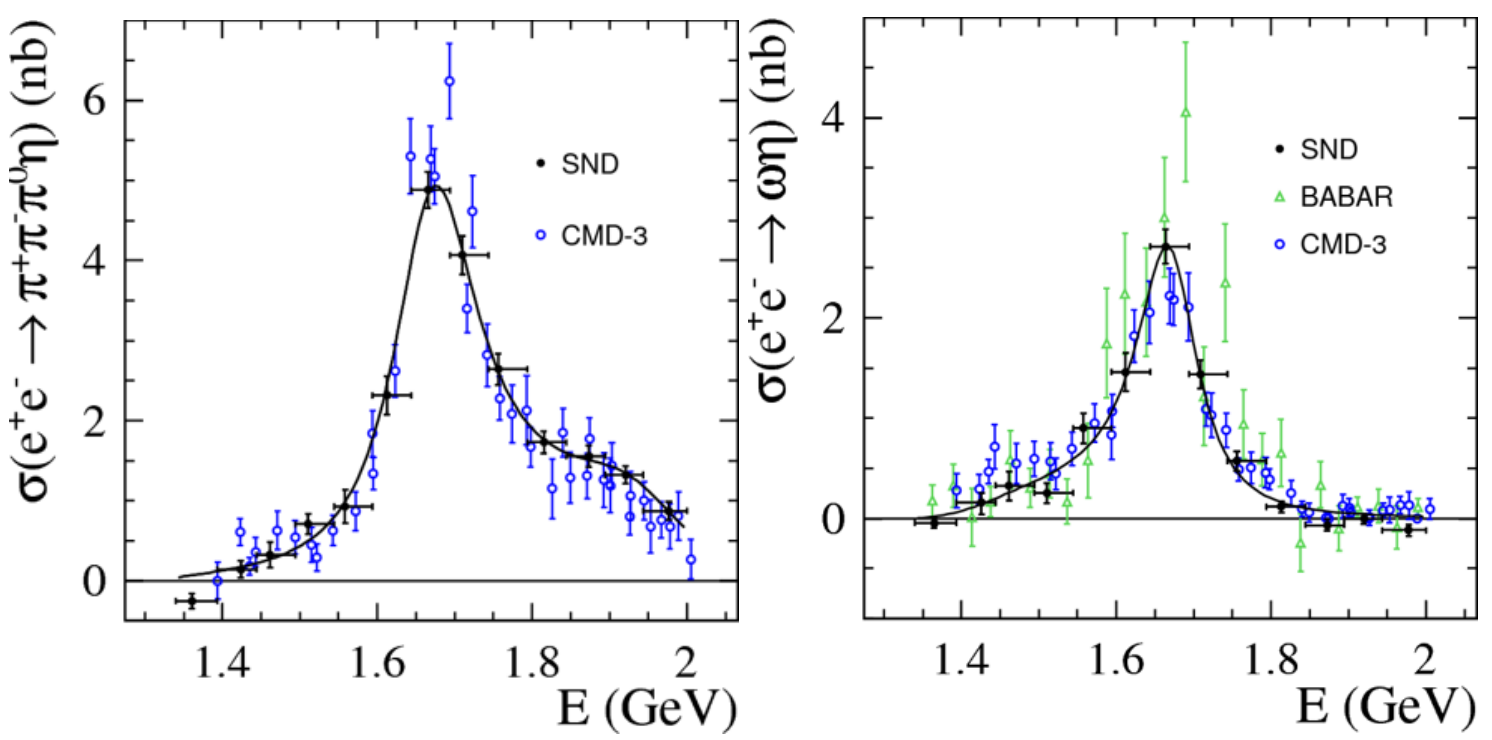

(a) The $e^{+} e^{-} \rightarrow \pi^{+} \pi^{-} \pi^{0} \eta$ cross section measured (b) The $e^{+} e^{-} \rightarrow \omega \eta$ cross section measured by SND by SND

in comparison with BABAR data [2], [9]]. The curve is the result of the VMD fit

Figure 7

Our results disagree with the BABAR data at $E>1.6 \mathrm{GeV}$. The fit to the cross section was performed by a sum of two resonances: $\omega(1420)$ and an effective resonance describing $\omega(1650)$ and $\phi(1680)$ contributions. The cross section for the subprocess $e^{+} e^{-} \rightarrow \phi \eta$ in comparison with the BABAR data [22] is shown in Fig. [8a. The total cross section for $e^{+} e^{-} \rightarrow a^{0} \rho$ and structureless components in comparison with CMD-3 data[ए]] is shown in Fig.

Process $e^{+} e^{-} \rightarrow \omega \pi^{0}$

Preliminary results for cross section of the $e^{+} e^{-} \rightarrow \omega \pi^{0} \rightarrow \pi^{+} \pi^{-} \pi^{0} \pi^{0}$ process in the energy range $1-2 \mathrm{GeV}$ in comparison with previous SND measurements [ [22], 24, 25] are presented in Fig.Q. The statistical error varies between 2 and 16\%, while systemaic errors are between 1 and $9 \%$ depending on the energy. The cross section is fitted using contributions of the $\rho, \rho(1450)$ and $\rho(1700)$ resonances. 

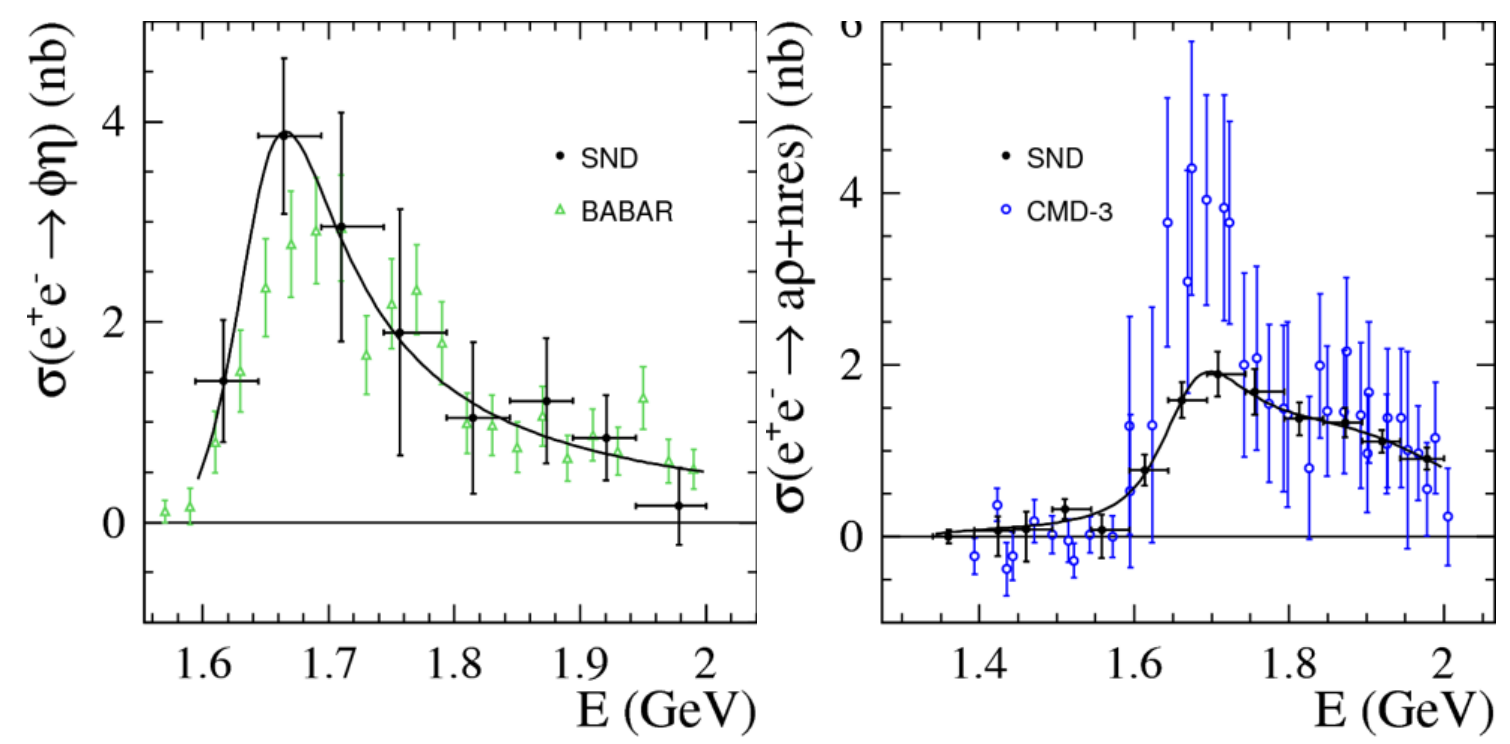

(a) The $e^{+} e^{-} \rightarrow \phi \eta$ cross section measured by SND (b) The total cross section for $e^{+} e^{-} \rightarrow a^{0} \rho$ and in comparison with BABAR data [22]. The curve is structureless components in comparison with CMDthe result of the VMD fit 3 data[며]

Figure 8

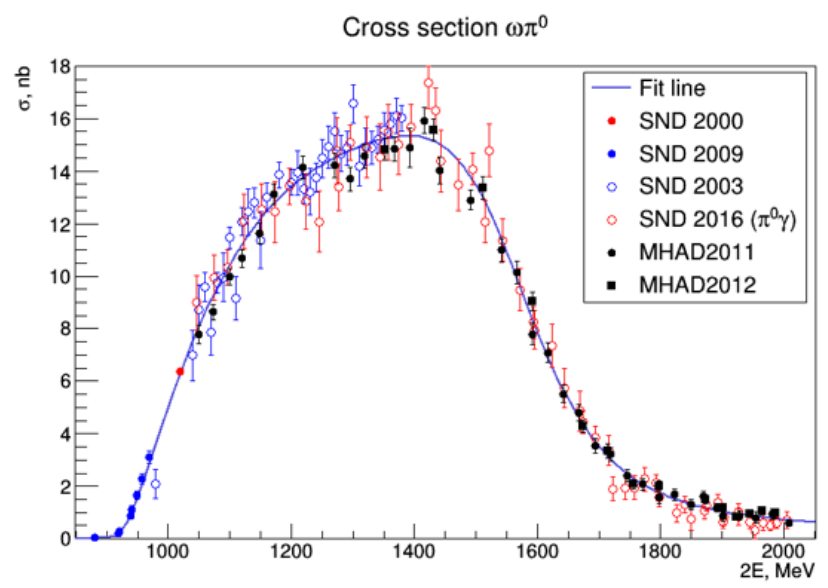

Figure 9: The $e^{+} e^{-} \rightarrow \omega \pi^{0} \rightarrow \pi^{+} \pi^{-} \pi^{0} \pi^{0}$ cross section measured by SND at VEPP-2000 and VEPP-2M ([24, [25]) in comparison with $e^{+} e^{-} \rightarrow \omega \pi^{0} \rightarrow \pi^{0} \pi^{0} \gamma$ [23] cross section.

Process $e^{+} e^{-} \rightarrow \pi^{0} \gamma$

The $e^{+} e^{-} \rightarrow \pi^{0} \gamma$ cross section is the third largest cross section (after $e^{+} e^{-} \rightarrow \pi^{+} \pi^{-}$and $\pi^{+} \pi^{-} \pi^{0}$ ) below $1 \mathrm{GeV}$. From analysis of the $e^{+} e^{-} \rightarrow \pi^{0} \gamma$ data in the vector meson dominance (VMD) model, the widths of vector-meson radiative decays are extracted, which are widely used in phenomenological models. The most accurate data on this process were obtained in experiments at

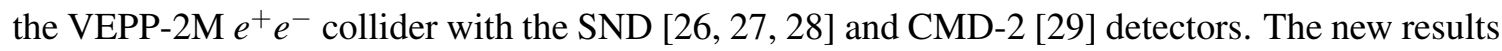
[BO] based on VEPP-2000 data for two energy regions is presented in Figs. 10a],

In the energy range $1.1-1.4 \mathrm{GeV}$ we see the contribution of the $\omega(1420)$ and $\rho(1450)$ reso- 
nances. The cross section in the energy region $1.4-2.0 \mathrm{GeV}$ is small indicating that the probabilities of the decays to $\pi^{0} \gamma$ of $\omega(1680)$ and $\rho(1700)$ are small.
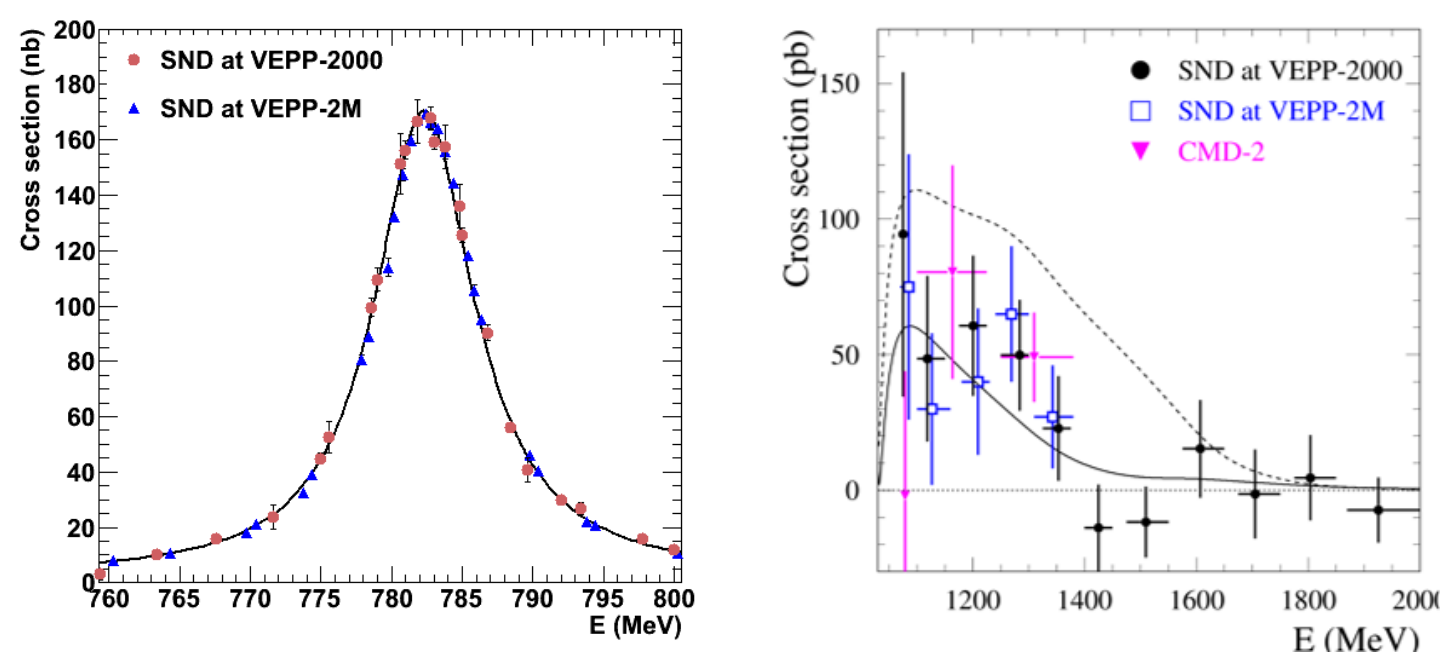

(a) The $e^{+} e^{-} \rightarrow \pi^{0} \gamma$ cross section in the $\rho-\omega$ mass (b) The $e^{+} e^{-} \rightarrow \pi^{0} \gamma$ cross section in the energy energy range measured by SND at VEPP-2000 in range above $\phi$-resonance mass measured by SND comparison with previous SND result [R6] at VEPP-2000 in comparison with previous SND [R6] and CMD-2 [2Q] results

Figure 10

Process $e^{+} e^{-} \rightarrow \pi^{+} \pi^{-} 4 \pi^{0}$

We present very preliminary result of the process $e^{+} e^{-} \rightarrow \pi^{+} \pi^{-} 4 \pi^{0}$ cross section (Fig. $\square$ ). This process was never studied before. Final states with other isospin combinations $\left(4 \pi^{+} 4 \pi^{-}\right.$and $2 \pi^{+} 2 \pi^{-} 2 \pi^{0}$ ) are known quite well (BaBar and CMD-3) and show clear structure near the protons and neutrons production threshold [B], B2]

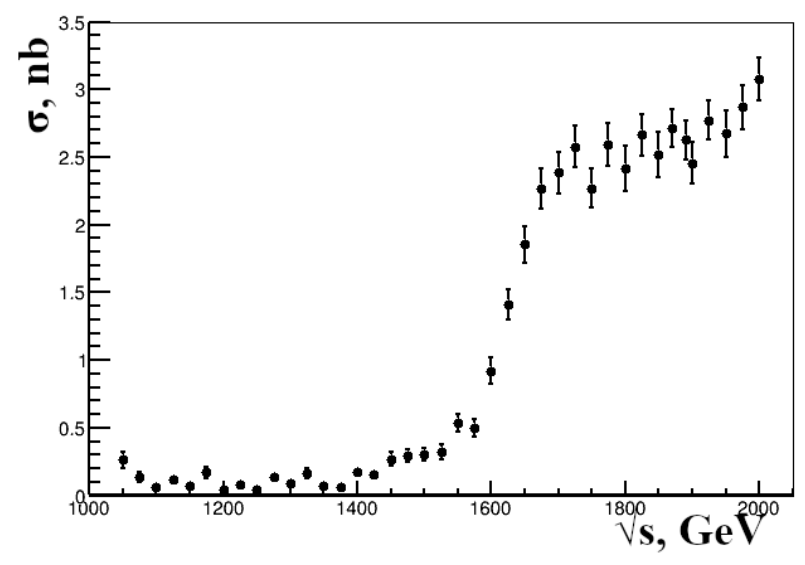

Figure 11: The $e^{+} e^{-} \rightarrow \pi^{+} \pi^{-} 4 \pi^{0}$ cross section in the energy region $1.05-2 \mathrm{GeV}$ 


\section{Conclusions}

During $2010-2013$ the SND detector accumulated $\sim 70 \mathrm{pb}^{-1}$ of integrated luminosity at the VEPP-2000 electron-positron collider in the c.m. energy range $0.3-2 \mathrm{GeV}$. After VEPP2000 and SND upgrade during $2014-2016$ the data taking runs started again and $\sim 120 \mathrm{pb}^{-1}$ of integrated luminosity is already collected. Data analysis on hadron production is partly published but more is still in progress. The obtained results have comparable or better accuracy than previous measurements. For some processes the cross sections have been measured for the first time.

\section{Acknowledgments}

This work is supported in part by the RFBR grants 16-02-00327, 16-02-00014, 18-02-00382-a, 18-02-00147-a. Part of this work related to the beam energy measurement and the photon reconstruction algorithm in the electromagnetic calorimeter for multiphoton events is supported by the Russian Science Foundation (project No. 14-50-00080).

\section{References}

[1] M. N. Achasov et al., Spherical neutral detector for VEPP-2M collider, Ducl._nstrum. Meth.A449 (2000)125 [hep-ex/9909015].

[2] G. N. Abramov et al., SND upgrade, eConf C010430 (2001) T10 [hep-ex/0105093].

[3] CMD-3, SND collaboration, B. I. Khazin, Detectors and physics at VEPP-2000, Ducl. Instrum Meth. A623 (2010) 353.

[4] V. M. Aulchenko et al., SND tracking system: Tests with cosmic muons, Wucl._nstrum. Meth. A598 (2009)102

[5] E. V. Abakumova et al., A system of beam energy measurement based on the Compton backscattered laser photons for the VEPP-2000 electron-positron collider, Nucl. Instrum. Meth. A744(2014) 35 [13]0.7764].

[6] P. Yu. Shatunov et al., Status and perspectives of the VEPP-2000, Phys. Part. Nucl. Lett. 13 (2016) 995.

[7] M. N. Achasov and A. S. Kupich, Separation of $e^{+} e^{-} \rightarrow e^{+} e^{-}$and $e^{+} e^{-} \rightarrow \pi^{+} \pi^{-}$events based on the difference in the energy deposition profiles in SND detector calorimeter, DINST 12(2017) COO6035.

[8] BABAR collaboration, J. P. Lees et al., Precise Measurement of the $e^{+} e^{-} \rightarrow \pi^{+} \pi^{-}(\gamma)$ Cross Section with the Initial-State Radiation Method at BABAR, Phys. Rev. D86 (2012) 032013 [1205.2228].

[9] KLOE-2 collaboration, A. Anastasi et al., Combination of $\operatorname{KLOE} \sigma\left(e^{+} e^{-} \rightarrow \pi^{+} \pi^{-} \gamma(\gamma)\right)$ measurements and determination of $a_{\mu}^{\pi^{+}} \pi^{-}$in the energy range $0.10<s<0.95 \mathrm{GeV}^{2}$, $\mathrm{WHEP( \mathbb {3 }}$ 2018) 173 [1]71].03085].

[10] SND collaboration, M. N. Achasov et al., Search for the process $\mathbf{e}^{+} \mathbf{e}^{-} \rightarrow \eta$, Phys. Rev. D98(2018) 052007 [1806.07609].

[11] M. N. Achasov et al., Study of the process $e^{+} e^{-} \rightarrow n \bar{n}$ at the VEPP-2000 $e^{+} e^{-}$collider with the SND detector, Phys. Rev. D90 (2014) 112007] [14 410.3188]]. 
[12] M. N. Achasov et al., Measurement of the $e^{+} e \rightarrow \eta K^{+} K$ Cross Section by Means of the SND Detector, Phys. Atom. Nucl. 81 (2018) 205.

[13] BABAR collaboration, B. Aubert et al., Measurements of $e^{+} e^{-} \rightarrow K^{+} K^{-} \eta, K^{+} K^{-} \pi^{0}$ and $K_{s}^{0} K^{ \pm} \pi^{\mp}$ cross- sections using initial state radiation events, Phys. Rev. D77(2008) 092002 [0710.445]].

[14] M. N. Achasov et al., Measurement of the $e^{+} e^{-} \rightarrow \eta \pi^{+} \pi^{-}$cross section with the SND detector at the VEPP-2000 collider, Phys. Rev. D97(2018) 012008 [ [1] [1].08862]].

[15] SND collaboration, V. M. Aulchenko et al., Measurement of the $e^{+} e^{-} \rightarrow \eta \pi^{+} \pi^{-}$cross section in the center-of-mass energy range 1.22-2.00 GeV with the SND detector at the VEPP-2000 collider, Phys. Rev. D91(2015) 052013 [1412.1971].

[16] S. Godfrey and N. Isgur, Mesons in a Relativized Quark Model with Chromodynamics, Phys. Rev. D32 (1985) 189.

[17] M. N. Achasov et al., Measurement of the $\mathbf{e}^{+} \mathbf{e}^{-} \rightarrow \mathbf{K}_{\mathbf{S}} \mathbf{K}_{\mathbf{L}} \pi^{0}$ cross section in the energy range $\sqrt{\mathbf{s}}=1.3-2.0 \mathrm{GeV}$, Phys. Rev. D97(2018)03201] [1]71. 07143].

[18] BABAR collaboration, J. P. Lees et al., Cross sections for the reactions $e^{+} e^{-} \rightarrow K_{S}^{0} K_{L}^{0} \pi^{0}, K_{S}^{0} K_{L}^{0} \eta$, and $K_{S}^{0} K_{L}^{0} \pi^{0} \pi^{0}$ from events with initial-state radiation, Phys. Rev. D95 (2017) 052001] [1] 70]. 0829]].

[19] CMD-3 collaboration, R. R. Akhmetshin et al.Phys.Lett. B773 (2017) 150.

[20] SND collaboration, M. N. Achasov et al.Phys. Rev. D94 (2016) 092002.

[21] BABAR collaboration, B. Aubert et al.Phys. Rev. D73 (2006) 052003.

[22] BABAR collaboration, B. Aubert et al.Phys. Rev. D76 (2007) 092005.

[23] SND collaboration, M. N. Achasov et al.Phys. Rev. D94 (2016) 112001.

[24] SND collaboration, M. N. Achasov et al.J. Exp. Theor. Phys. 96 (2003) 789.

[25] SND collaboration, M. N. Achasov et al.J. Exp. Theor. Phys. 109 (2009) 379.

[26] SND collaboration, M. N. Achasov et al.Phys. Rev. D93 (2016) 092001.

[27] SND collaboration, M. N. Achasov et al.Eur.Phys.J. C12 (2000) 25.

[28] SND collaboration, M. N. Achasov et al.Phys.Lett. B559 (2003) 171.

[29] CMD-2 collaboration, R. R. Akhmetshin et al.Phys.Lett. B605, 26 (2005) .

[30] M. N. Achasov et al., Measurement of the $e^{+} e^{-} \rightarrow \pi^{0} \gamma$ cross section in the energy range 1.075-2 GeV at SND, 1809.07631.

[31] BABAR collaboration, B. Aubert et al., The $e^{+} e^{-} \rightarrow 3\left(\pi^{+} \pi^{-}\right), 2\left(\pi^{+} \pi^{-} \pi^{0}\right)$ and $K^{+} K^{-} 2\left(\pi^{+} \pi^{-}\right)$ cross sections at center-of-mass energies from production threshold to 4.5-GeV measured with initial-state radiation, Phys. Rev. D73 (2006) 052003 [hep-ex/0602006].

[32] P. A. Lukin et al., Investigation of the processes $e^{+} e \rightarrow 2\left(\pi^{+} \pi \pi^{0}\right)$ and $e^{+} e \rightarrow 3\left(\pi^{+} \pi\right)$ with the aid of the CMD-3 detector, Phys. Atom. Nucl. 78 (2015) 353. 